\title{
Combinatorics of linear iterated function systems with overlaps
}

\author{
Nikita Sidorov \\ School of Mathematics, The University of Manchester, PO Box 88, Sackville Street, \\ Manchester M60 1QD, UK \\ E-mail: sidorov@manchester.ac.uk
}

Received 10 December 2006, in final form 20 March 2007

Published 17 April 2007

Online at stacks.iop.org/Non/20/1299

Recommended by C P Dettmann

Abstract

Let $\boldsymbol{p}_{0}, \ldots, \boldsymbol{p}_{m-1}$ be points in $\mathbb{R}^{d}$, and let $\left\{f_{j}\right\}_{j=0}^{m-1}$ be a one-parameter family of similitudes of $\mathbb{R}^{d}$ :

$$
f_{j}(\boldsymbol{x})=\lambda \boldsymbol{x}+(1-\lambda) \boldsymbol{p}_{j}, \quad j=0, \ldots, m-1,
$$

where $\lambda \in(0,1)$ is our parameter. Then, as is well known, there exists a unique self-similar attractor $S_{\lambda}$ satisfying $S_{\lambda}=\bigcup_{j=0}^{m-1} f_{j}\left(S_{\lambda}\right)$. Each $\boldsymbol{x} \in S_{\lambda}$ has at least one address $\left(i_{1}, i_{2}, \ldots\right) \in \prod_{1}^{\infty}\{0,1, \ldots, m-1\}$, i.e. $\lim _{n} f_{i_{1}} f_{i_{2}} \ldots f_{i_{n}}(\mathbf{0})=\boldsymbol{x}$.

We show that for $\lambda$ sufficiently close to 1 , each $\boldsymbol{x} \in S_{\lambda} \backslash\left\{\boldsymbol{p}_{0}, \ldots, \boldsymbol{p}_{m-1}\right\}$ has $2^{\aleph_{0}}$ different addresses. If $\lambda$ is not too close to 1 , then we can still have an overlap, but there exist $x$ s which have a unique address. However, we prove that almost every $\boldsymbol{x} \in S_{\lambda}$ has $2^{\aleph_{0}}$ addresses, provided $S_{\lambda}$ contains no holes and at least one proper overlap. We apply these results to the case of expansions with deleted digits.

Furthermore, we give sharp sufficient conditions for the open set condition to fail and for the attractor to have no holes.

These results are generalizations of the corresponding one-dimensional results, however most proofs are different.

Mathematics Subject Classification: 60J05

\section{One-dimensional case: an overview}

The purpose of this paper is to generalize certain results concerning a one-dimensional model considered in $[7,13,14]$, so we first describe this model. Let $\lambda \in(1 / 2,1)$ be our parameter. Consider a pair of similitudes of $I=[0,1]$ :

$$
\begin{aligned}
& f_{0}(x)=\lambda x, \\
& f_{1}(x)=\lambda x+1-\lambda .
\end{aligned}
$$


They constitute an iterated function system (IFS). More precisely, choose 0 as a starting point, and for any sequence $\left(\varepsilon_{1}, \varepsilon_{2}, \ldots\right)$ of 0 s and $1 \mathrm{~s}$ :

$$
x=\lim _{N \rightarrow+\infty} f_{\varepsilon_{1}} \ldots f_{\varepsilon_{N}}(0),
$$

a forward iteration (the limit is independent of our choice of the starting point). The set of all $x \mathrm{~s}$ that are representable in such a form is called the attractor of an IFS. As is well known, in this case $\lambda>1 / 2$ yields the attractor $[0,1]$.

Unlike a general IFS, in this model any composition of $f_{0}$ and $f_{1}$ can be given in a very simple form:

$$
f_{\varepsilon_{1}} \ldots f_{\varepsilon_{N}}(0)=\left(\lambda^{-1}-1\right) \sum_{n=1}^{N} \varepsilon_{n} \lambda^{n}
$$

whence

$$
x=\lim _{N \rightarrow \infty} \sum_{n=1}^{N} \varepsilon_{k} \lambda^{n}=\left(\lambda^{-1}-1\right) \sum_{n=1}^{\infty} \varepsilon_{n} \lambda^{n}
$$

(sometimes called a $\lambda$-expansion of $x$ ). Since $\lambda>1 / 2$, we have a proper overlap

$$
f_{0}(I) \cap f_{1}(I)=[1-\lambda, \lambda],
$$

so one might expect a typical $x \in(0,1)$ to have infinitely many distinct $\lambda$-expansions (= addresses $)\left(\varepsilon_{1}, \varepsilon_{2}, \ldots\right) \in \prod_{1}^{\infty}\{0,1\}$-which indeed proves to be the case.

More precisely, put

$$
\mathfrak{R}_{\lambda}(x)=\left\{\left(\varepsilon_{n}\right)_{1}^{\infty}: x=\left(\lambda^{-1}-1\right) \sum_{n=1}^{\infty} \varepsilon_{n} \lambda^{n}\right\} .
$$

The following important result regarding $\mathfrak{R}_{\lambda}(x)$ has been obtained by Erdôs, Joó and Komornik.

Theorem 1.1 [5]. If $\lambda>g=\frac{\sqrt{5}-1}{2}=0.618 \ldots$, then $\mathfrak{R}_{\lambda}(x)$ has the cardinality of the continuum for each $x \in(0,1)$.

Moreover, the golden ratio $g$ proves to be a sharp constant in the previous theorem. Nonetheless, the following metric result holds for $\lambda \in(1 / 2, g]$.

Theorem 1.2 [13]. For any $\lambda \in(1 / 2, g]$ the cardinality of $\mathfrak{R}_{\lambda}(x)$ is the continuum for Lebesgue-a.e. $x \in(0,1)$.

Put

$$
\mathcal{U}_{\lambda}=\left\{x \in(0,1) \mid !\left(\varepsilon_{n}\right)_{1}^{\infty}: x=\left(\lambda^{-1}-1\right) \sum_{n=1}^{\infty} \varepsilon_{n} \lambda^{n}\right\}
$$

(the set of uniqueness). By theorem $1, \mathcal{U}_{\lambda}=\emptyset$ if $\lambda>g$.

Theorem 1.3 [7]. The set $\mathcal{U}_{\lambda}$ is

- countable for $\lambda \in\left(\lambda_{*}, g\right)$;

- uncountable for zero Hausdorff dimension if $\lambda=\lambda_{*}$; and

- a set of positive Hausdorff dimension for $\lambda \in\left(1 / 2, \lambda_{*}\right)$.

Here $\lambda_{*}=0.559525 \ldots$ denotes the (transcendental) Komornik-Loreti constant introduced in [9].

The purpose of this paper is to generalize some of these results to linear IFSs in higher dimensions. 


\section{Multidimensional case: an analogue of theorem 1.1}

Let $\boldsymbol{p}_{0}, \ldots, \boldsymbol{p}_{m-1}$ be distinct points in $\mathbb{R}^{d}$, and let $\left\{f_{j}\right\}_{j=0}^{m-1}$ be a one-parameter family of similitudes of $\mathbb{R}^{d}$ :

$$
f_{j}(\boldsymbol{x})=\lambda \boldsymbol{x}+(1-\lambda) \boldsymbol{p}_{j}, \quad j=0, \ldots, m-1,
$$

where $\lambda \in(0,1)$ is our parameter ${ }^{1}$.

Then, as is well known, there exists a unique self-similar attractor $S_{\lambda}$ satisfying

$$
S_{\lambda}=\bigcup_{j=0}^{m-1} f_{j}\left(S_{\lambda}\right) .
$$

Put $\mathcal{A}=\{0, \ldots, m-1\}$. Similarly to the one-dimensional model, every $\boldsymbol{x} \in S_{\lambda}$ has at least one address, i.e. a sequence $\left(i_{1}, i_{2}, \ldots\right) \in \mathcal{A}^{\mathbb{N}}$ such that

$$
\begin{aligned}
\boldsymbol{x} & =\lim _{n \rightarrow+\infty} f_{i_{1}} \ldots f_{i_{n}}\left(\boldsymbol{x}_{0}\right) \\
& =\left(\lambda^{-1}-1\right) \sum_{n=1}^{\infty} \lambda^{n} \boldsymbol{p}_{i_{n}},
\end{aligned}
$$

where $\boldsymbol{x}_{0} \in \mathbb{R}^{d}$ is arbitrary. We assume the dimension of the convex hull of $\left\{\boldsymbol{p}_{0}, \ldots, \boldsymbol{p}_{m-1}\right\}$ to be equal to $d$. (Otherwise we embed $S_{\lambda}$ into $\mathbb{R}^{d^{\prime}}$ with $d^{\prime}<d$.)

Recall that an IFS is said to satisfy the open set condition (OSC) if there exists an open set $O \subset \mathbb{R}^{d}$ such that

$$
O=\bigcup_{j=0}^{m-1} f_{j}(O),
$$

with the union being disjoint. Loosely speaking, the OSC means that the images $f_{j}(\Omega)$ do not intersect properly, where $\Omega$ is the convex hull of the $\boldsymbol{p}_{j}$. Virtually all famous IFS-generated fractals (the Sierpiński gasket, Sierpiński carpet, von Koch curve, etc) originate from IFSs that satisfy the OSC.

We will be interested in IFSs which do not satisfy the OSC. Here is a simple sufficient condition.

Proposition 2.1. If $\lambda>m^{-1 / d}$, then the OSC is not satisfied.

Proof. Assume there exists an open set $O$ which satisfies the definition. Since $f_{j}(O) \subset O$ and the images are disjoint, we have $f_{i} f_{j}(O) \cap f_{i^{\prime}} f_{j^{\prime}}(O)=\varnothing$ if $(i, j) \neq\left(i^{\prime}, j^{\prime}\right)$, and by induction, $f_{i_{1}} \ldots f_{i_{n}}(O) \cap f_{j_{1}} \ldots f_{j_{n}}(O)=\emptyset$ provided $\left(i_{1}, \ldots, i_{n}\right) \neq\left(j_{1}, \ldots, j_{n}\right)$.

Since $\mathcal{L}_{d}\left(f_{i_{1}} \ldots f_{i_{n}}(O)\right)=\lambda^{d n} \mathcal{L}_{d}(O)$ (where $\mathcal{L}_{d}$ denotes the $d$-dimensional Lebesgue measure), and the number of different words of length $n$ is $m^{n}$, the pigeonhole principle yields a contradiction with $\lambda>m^{-1 / d}$.

Example 2.2. Let $\boldsymbol{p}_{0}, \boldsymbol{p}_{1}, \boldsymbol{p}_{2}$ be three noncollinear points in $\mathbb{R}^{2}$-vertices of a triangle $\Delta$. Consider the IFS

$$
f_{j}(\boldsymbol{x})=\lambda \boldsymbol{x}+(1-\lambda) \boldsymbol{p}_{j}, \quad j=0,1,2,
$$

1 To simplify our notation, we have decided to avoid notation like $f_{j}^{(\lambda)}$, since there is never really any confusion regarding which $\lambda$ is being considered at a given moment. 
and, following [1], we denote the attractor by $\mathcal{S}_{\lambda}$, i.e.

$$
\mathcal{S}_{\lambda}=\bigcup_{j=0}^{2} f_{j}\left(\mathcal{S}_{\lambda}\right)
$$

Note that for $\lambda=1 / 2$ the set $\mathcal{S}_{\lambda}$ is the famous Sierpiński gasket. If $\lambda \leqslant 1 / 2$, then the IFS does satisfy the OSC, and for $\lambda \geqslant 2 / 3$ we have $\mathcal{S}_{\lambda}=\Delta$, i.e. $\mathcal{S}_{\lambda}$ contains no holes. If $\lambda>1 / 2$, then we have a proper overlap, i.e. $f_{i}(\Delta) \cap f_{j}(\Delta)$ has a nonempty interior.

Remark 2.3. For the triangular case a more delicate argument allows one to show that $\lambda>1 / 2$ implies the failure of the OSC (instead of $\lambda>1 / \sqrt{3}$ provided by proposition 2.1 )— see [1, proposition 3.9].

Return to the general case. Put

$$
\Omega=\operatorname{conv}\left(\boldsymbol{p}_{0}, \ldots, \boldsymbol{p}_{m-1}\right) .
$$

Clearly, $S_{\lambda} \subset \Omega$. We give a universal sufficient condition for $\Omega$ to have no holes.

Proposition 2.4. If $\lambda \geqslant d /(d+1)$, then $S_{\lambda}=\Omega$, i.e. our attractor has no holes.

Proof. Let $\boldsymbol{p}_{0}, \ldots, \boldsymbol{p}_{k-1}$ be the vertices of $\Omega$ (with $k \leqslant m$ ) and let $\mathcal{F}_{1}, \ldots, \mathcal{F}_{h}$ denote its $(d-1)$-dimensional faces. Note that $k \geqslant d+1$, and if $k=d+1$, we have a simplex for which the claim is proved in [1]. Assume $k>d+1$.

For $\boldsymbol{x} \in \Omega$ we denote its distance to $\mathcal{F}_{i}$ by $x_{i}$. Then adding the volumes of the pyramids with the vertex $\boldsymbol{x}$ and the bases $\mathcal{F}_{i}$ yields

$$
\frac{1}{d} \sum_{i=1}^{h} \mathcal{L}_{d-1}\left(\mathcal{F}_{i}\right) \cdot x_{i}=\mathcal{L}_{d}(\Omega) .
$$

It suffices to show that $\Omega=\bigcup_{j=0}^{k-1} f_{j}(\Omega)$. Assume, on the contrary, that there exists $\boldsymbol{x} \in \Omega \backslash \bigcup_{j=0}^{k-1} f_{j}(\Omega)$. Since $f_{j}(\Omega)=\lambda \Omega+(1-\lambda) \boldsymbol{p}_{j}$, we have

$$
\frac{x_{i}}{\operatorname{dist}\left(\boldsymbol{p}_{j}, \mathcal{F}_{i}\right)}<1-\lambda, \quad i=1, \ldots, h,
$$

for all $j \in\{0, \ldots, k-1\}$ such that $\boldsymbol{p}_{j} \notin \mathcal{F}_{i}$. Put

$$
\alpha_{i}=\max _{0 \leqslant j \leqslant k-1} \operatorname{dist}\left(\boldsymbol{p}_{j}, \mathcal{F}_{i}\right) \quad i=1, \ldots, h .
$$

Then by (2.2) and (2.3),

$$
\lambda<1-\frac{d \mathcal{L}_{d}(\Omega)}{\sum_{i=1}^{h} \alpha_{i} \mathcal{L}_{d-1}\left(\mathcal{F}_{i}\right)} .
$$

To complete the proof, it suffices to show that

$$
\sum_{i=1}^{h} \alpha_{i} \mathcal{L}_{d-1}\left(\mathcal{F}_{i}\right) \leqslant d(d+1) \mathcal{L}_{d}(\Omega) .
$$

Consider the family of affine copies of $\Omega$ with the ratio $d /(d+1)$, i.e.

$$
\left\{\frac{d}{d+1} \Omega+\frac{1}{d} \boldsymbol{p}_{j} \mid j \in\{0,1, \ldots, k-1\}\right\} .
$$


Let $0 \leqslant j_{1}<\ldots<j_{d+1} \leqslant k-1$ and let $\Delta_{j_{1} \ldots j_{d+1}}=\operatorname{conv}\left(\boldsymbol{p}_{j_{1}}, \ldots, \boldsymbol{p}_{j_{d+1}}\right)$, a $d$-dimensional simplex. We have

$$
\bigcap_{r=1}^{d+1}\left(\frac{d}{d+1} \Delta_{j_{1} \ldots j_{d+1}}+\frac{1}{d} \boldsymbol{p}_{j_{r}}\right) \neq \varnothing
$$

(the intersection contains the centre of mass of $\Delta_{j_{1} \ldots j_{d+1}}$ ), whence

$$
\bigcap_{r=1}^{d+1}\left(\frac{d}{d+1} \Omega+\frac{1}{d} \boldsymbol{p}_{j_{r}}\right) \neq \varnothing
$$

for any $(d+1)$-tuple, as $\Delta_{j_{1} \ldots j_{d+1}} \subset \Omega$. Hence, by Helly's theorem (see, e.g., [4]), there exists

$$
z \in \bigcap_{j=0}^{k-1}\left(\frac{d}{d+1} \Omega+\frac{1}{d} \boldsymbol{p}_{j}\right)
$$

By our construction, the point $\boldsymbol{z}$ has the following property: if $\boldsymbol{y} \in \partial \Omega$ and $\boldsymbol{z} \in\left[\boldsymbol{p}_{j}, \boldsymbol{y}\right]$ for some $j \in\{0,1, \ldots, k-1\}$, then $|[\boldsymbol{z}, \boldsymbol{y}]| \geqslant \frac{1}{d+1}\left|\left[\boldsymbol{p}_{j}, \boldsymbol{y}\right]\right|$. Therefore, $\operatorname{dist}\left(\boldsymbol{p}_{j}, \mathcal{F}_{i}\right) \leqslant(d+1) \cdot \operatorname{dist}\left(\boldsymbol{z}, \mathcal{F}_{i}\right)$ for all $j \in\{0, \ldots, k-1\}$, whence by definition, $\alpha_{i} \leqslant(d+1) \cdot \operatorname{dist}\left(z, \mathcal{F}_{i}\right)$. Consequently,

$$
\sum_{i=1}^{h} \alpha_{i} \mathcal{L}_{d-1}\left(\mathcal{F}_{i}\right) \leqslant(d+1) \sum_{i=1}^{h} \operatorname{dist}\left(z, \mathcal{F}_{i}\right) \cdot \mathcal{L}_{d-1}\left(\mathcal{F}_{i}\right)
$$

and to obtain (2.4), it suffices to note that the volume of $\Omega$ equals the sum of the volumes of pyramids whose vertex is $z$, i.e.

$$
\mathcal{L}_{d}(\Omega)=\frac{1}{d} \sum_{i=1}^{h} \operatorname{dist}\left(z, \mathcal{F}_{i}\right) \cdot \mathcal{L}_{d-1}\left(\mathcal{F}_{i}\right)
$$

Lemma 2.5. Assume $\lambda$ is such that $S_{\lambda}=\Omega$ and suppose $i, j \in \mathcal{A}$ are such that $\Omega_{i j}:=$ $f_{i}(\Omega) \cap f_{j}(\Omega)$ has a nonempty interior.

Then each $\boldsymbol{x} \in \Omega_{i j}$ has at least one address beginning with $i$ and at least one address beginning with $j$.

Proof. Since $\boldsymbol{x} \in f_{i}(\Omega)$, there exists $\boldsymbol{x}^{\prime} \in \Omega$ such that $f_{i}\left(\boldsymbol{x}^{\prime}\right)=\boldsymbol{x}$. By our assumption, $\boldsymbol{x}^{\prime} \in S_{\lambda}$, whence $\boldsymbol{x}^{\prime}=\lim _{n} f_{i_{2}} \ldots f_{i_{n}}\left(\boldsymbol{x}_{0}\right)$. Therefore, in view of the continuity of $f_{i}$, we have $\boldsymbol{x}=\lim _{n} f_{i} f_{i_{2}} \ldots f_{i_{n}}\left(\boldsymbol{x}_{0}\right)$. The same argument applies to $j$.

Remark 2.6. The condition $S_{\lambda}=\Omega$, generally speaking, cannot be dropped; for instance, one can show that in the triangular case, if $\lambda \in(0.65,2 / 3)$, then we have a hole whose image under one of the maps lies in $\mathcal{S}_{\lambda}$ - see [1, proposition 3.7].

Theorem 2.7. For each $\boldsymbol{p}_{0}, \ldots, \boldsymbol{p}_{m-1}$ there exists $\lambda_{0}<1$ such that for any $\lambda \in\left(\lambda_{0}, 1\right)$

(a) there are no holes, i.e. $S_{\lambda}=\Omega$; and

(b) each point $\boldsymbol{x} \in \Omega$, except when $\boldsymbol{x}$ is a vertex of $\Omega$, has $2^{\aleph_{0}}$ distinct addresses.

Proof. Assume that $\lambda$ is large enough to ensure $S_{\lambda}=\Omega$. We will show that each $\boldsymbol{x}$ under consideration has a continuum of addresses $\left(i_{1}, i_{2}, \ldots\right) \in \mathcal{A}^{\prime}$, where $\mathcal{A}^{\prime}$ is the set of indices $i$ such that $\boldsymbol{p}_{i} \in \partial \Omega$. 
The idea of the proof is to use the multivalued inverse map $T_{\lambda}=\left\{f_{0}, \ldots, f_{m-1}\right\}^{-1}$. More precisely, put

$$
\Omega_{i}=f_{i}(\Omega) \backslash \bigcup_{j \neq i} f_{j}(\Omega)
$$

Clearly, if $\Omega_{i} \ni \boldsymbol{x} \sim\left(i_{1}, i_{2}, \ldots\right)$, then necessarily $i_{1}=i$. Conversely, if a point $\boldsymbol{x} \notin \Omega_{i}$ for any $i$, then by lemma 2.5 , there is a choice for the first symbol of its address.

Let $\boldsymbol{x} \in \Omega_{i} \backslash\left\{\boldsymbol{p}_{i}\right\}$; note that shifting its address $\left(i, i_{2}, i_{3}, \ldots\right)$ yields $\left(i_{2}, i_{3}, \ldots\right)$, which in $\Omega$ corresponds to applying $f_{i}^{-1}$. Let $\lambda_{0}$ be such that $f_{i}^{-1}\left(\Omega_{i}\right) \cap \Omega_{j}=\emptyset$ for all $i \neq j$ and all $\lambda>\lambda_{0}$.

Thus, by $f_{i}^{-1}$ being expanding on $\Omega_{i} \backslash\left\{\boldsymbol{p}_{i}\right\}$, we conclude that there exists $k \geqslant 1$ such that

$$
\begin{aligned}
& f_{i}^{-k}(\boldsymbol{x}) \in \Omega_{i}, \\
& \boldsymbol{x}^{\prime}=f_{i}^{-k-1}(\boldsymbol{x}) \in \Omega \backslash \bigcup_{i=0}^{m-1} \Omega_{i} .
\end{aligned}
$$

By our construction, $\boldsymbol{x}^{\prime}$ has at least two addresses; consider its two shifts, $\boldsymbol{x}^{\prime \prime}$ and $\boldsymbol{x}^{\prime \prime \prime}$, say. Either $\boldsymbol{x}^{\prime \prime} \in \Omega \backslash \bigcup_{i=0}^{m-1} \Omega_{i}$ and thus has at least two addresses itself or it belongs to $\Omega_{j}$ for some $j$ (and obviously, is not equal to $\boldsymbol{p}_{j}$ ) and, similarly to the above, we shift its address until it falls into $\Omega \backslash \bigcup_{i=0}^{m-1} \Omega_{i}$. Hence any $\boldsymbol{x} \in \Omega$ that is not one of its vertices has $2^{\aleph_{0}}$ distinct addresses.

Remark 2.8. Note that for the triangular case the sharp constant is $\lambda_{0} \approx 0.68233$, the unique positive root of $x+x^{3}=1$-see theorem 4.1 .

\section{Multidimensional case: generic behaviour}

\subsection{General theory: Lebesgue measure}

Let $U_{\lambda}$ denote the set of $\boldsymbol{x} \in S_{\lambda}$ having a unique address and $R_{\lambda}(\boldsymbol{x})$ denote the set of all addresses of a given $\boldsymbol{x} \in S_{\lambda}$.

Lemma 3.1. Put

$$
V_{\lambda}=\left\{x \in S_{\lambda}: \operatorname{card} R_{\lambda}(x)<2^{\aleph_{0}}\right\} .
$$

Then $\operatorname{dim}_{H} V_{\lambda}=\operatorname{dim}_{H} U_{\lambda}$ (where $\operatorname{dim}_{H}$ denotes Hausdorff dimension).

Proof. We are going to exploit the idea of branching introduced in [14]. Let $\boldsymbol{x} \in S_{\lambda}$ have at least two addresses; then there exists the smallest $n \geqslant 0$ such that $\boldsymbol{x} \sim\left(i_{1}, \ldots, i_{n}, i_{n+1}, \ldots\right)$ and $\boldsymbol{x} \sim\left(i_{1}, \ldots, i_{n}, i_{n+1}^{\prime}, \ldots\right)$ with $i_{n+1} \neq i_{n+1}^{\prime}$. We may depict this bifurcation as shown in figure 1 .

Assume that $\boldsymbol{x}$ has less than a continuum of distinct addresses. Then, inevitably, one of the branches at some point ceases to bifurcate. In other words, there exist $\left(i_{n}\right)_{1}^{\infty} \in \mathcal{A}^{\mathbb{N}}$ and $N \in \mathbb{N}$ such that $\boldsymbol{x} \sim\left(i_{1}, i_{2}, \ldots\right)$ and $\boldsymbol{x}^{\prime} \sim\left(i_{N}, i_{N+1}, \ldots\right) \in U_{\lambda}$. Hence

$$
V_{\lambda} \subset \bigcup_{\left(i_{1}, \ldots, i_{N}\right) \in \mathcal{A}^{N}} f_{i_{1}} \ldots f_{i_{N}}\left(U_{\lambda}\right) .
$$

Since the $f_{i}$ are linear, (3.1) implies $\operatorname{dim}_{H} V_{\lambda} \leqslant \operatorname{dim}_{H} U_{\lambda}$, and the inverse inequality is trivial. 


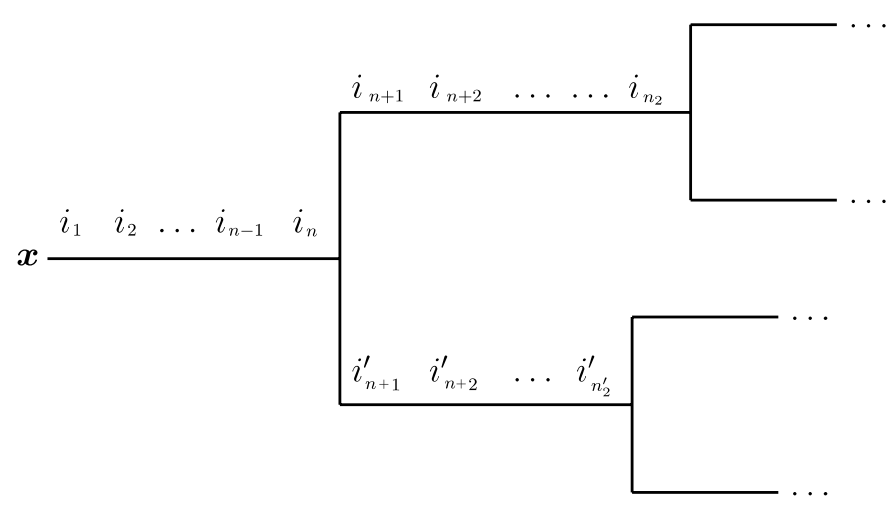

Figure 1. Branching and bifurcations.

Remark 3.2. Note that if $\lambda_{0}$ in theorem 2.7 is sharp, we always have a nonempty set of uniqueness for $\lambda<\lambda_{0}$, because, as we know from the branching argument, the existence of $\boldsymbol{x}$ with less than a continuum of addresses implies the existence of $\boldsymbol{x}^{\prime}$ with a unique address. For an example see section 4 .

Our goal is to show that, similarly to the one-dimensional case, if there are no holes and at least one proper overlap, then a.e. $\boldsymbol{x}$ has a continuum of addresses. We need an auxiliary claim from dimension theory.

Lemma 3.3. Let $A \subset \mathbb{R}^{d}$ be such that there exists a positive constant $\delta>0$ such that for an arbitrary cube $\mathcal{C} \subset \mathbb{R}^{d}$ which intersects $A$, one can find a cube $\mathcal{C}_{0} \subset C$ such that $\mathcal{L}_{d}\left(\mathcal{C}_{0}\right) \geqslant \delta \mathcal{L}_{d}(\mathcal{C})$ and $\mathcal{C}_{0} \cap A=\emptyset$.

Then $\operatorname{dim}_{H} A<d$.

Proof. Since $\operatorname{dim}_{H} A \leqslant \operatorname{dim}_{B} A$ (lower box-counting dimension), it suffices to show that $\underline{\operatorname{dim}}_{B} A<d$. Recall that there are various definitions of $\underline{\operatorname{dim}}_{B}$ (see [6, chapter 3.1]) and in particular, one which involves mesh cubes which we will use.

More precisely, a cube of the form $\left[m_{1} \varepsilon,\left(m_{1}+1\right) \varepsilon\right] \times \ldots \times\left[m_{d} \varepsilon,\left(m_{d}+1\right) \varepsilon\right]$, for some $\varepsilon>0$ and $m_{1}, \ldots, m_{d}$ integers, is called an $\varepsilon$-mesh cube. Let $\mathcal{N}_{\varepsilon}(A)$ denote the number of $\varepsilon$-mesh cubes which intersect $A$. Then

$$
\underline{\operatorname{dim}}_{B} A=\liminf _{\varepsilon \rightarrow 0} \frac{\log \mathcal{N}_{\varepsilon}(A)}{\log (1 / \varepsilon)} .
$$

It is obvious that our condition implies that there exists $M \in \mathbb{N}$ such that for any $\varepsilon$-mesh cube $C$ which intersects $A$, there exists an $\varepsilon / M$-mesh cube $C_{0} \subset C$ which does not. Hence

$$
\mathcal{N}_{\varepsilon / M}(A) \leqslant\left(M^{d}-1\right) \mathcal{N}_{\varepsilon}(A)
$$

which implies

$$
\mathcal{N}_{M^{-n}}(A) \leqslant\left(M^{d}-1\right)^{n} \cdot \mathcal{N}_{1}(A) .
$$

Consequently,

$$
\liminf _{\varepsilon \rightarrow 0} \frac{\log \mathcal{N}_{\varepsilon}(A)}{\log 1 / \varepsilon} \leqslant \frac{\log \left(M^{d}-1\right)}{\log M}<d .
$$

Now we are ready to prove a key technical lemma. 
Lemma 3.4. Let $\left\{F_{j}\right\}_{i=0}^{L-1}$ be a finite family of linear contractions of $\mathbb{R}^{d}$ with the same contraction ratio $\beta \in(0,1)$. Let $\Omega \subset \mathbb{R}^{d}$ be a polyhedron of dimension $d$, and assume $F_{j}(\Omega) \subset \Omega$ for all $j=0, \ldots, L-1$, and $\Omega=\bigcup_{j=0}^{L-1} F_{j}(\Omega)$ (no holes).

Put

$$
W_{n}=\bigcup_{\substack{\left(j_{1}, \ldots, j_{n}\right) \in\{0, \ldots, L-1\}^{n}: \\ \exists \in \in\{1, \ldots, n\}: j_{k}=0}} F_{j_{1}} \ldots F_{j_{n}}(\Omega) .
$$

Then $W_{n} \subset W_{n+1}$ for all $n \geqslant 1$, and $\mathcal{L}_{d}(\Omega \backslash W)=0$, where $W=\bigcup_{n \geqslant 1} W_{n}$. Furthermore, $\operatorname{dim}_{H}(\Omega \backslash W)<d$.

Proof. Our first goal is to show that $W_{n} \subset W_{n+1}$ for all $n \geqslant 1$. Since $\Omega=\bigcup_{j=0}^{L-1} F_{j}(\Omega)$, we have by induction,

$$
\bigcup_{\left(j_{1}, \ldots, j_{k}\right) \in\{0, \ldots, L-1\}^{k}} F_{j_{1}} \ldots F_{j_{k}}(\Omega)=\Omega
$$

whence for any $k<n$,

$$
\bigcup_{\left(j_{1}, \ldots, j_{n}\right) \in\{0, \ldots, L-1\}^{n}} F_{j_{1}} \ldots F_{j_{k}} F_{0} F_{j_{k+1}} \ldots F_{j_{n}}(\Omega)=\bigcup_{\left(j_{1}, \ldots, j_{k}\right) \in\{0, \ldots, L-1\}^{k}} F_{j_{1}} \ldots F_{j_{k}} F_{0}(\Omega) .
$$

Hence by definition, $W_{1}=F_{0}(\Omega)$ and

$$
W_{n}=\left(\bigcup_{k=1}^{n-1} \bigcup_{\left(j_{1}, \ldots, j_{k}\right) \in\{0, \ldots, L-1\}^{k}} F_{j_{1}} \ldots F_{j_{k}} F_{0}(\Omega)\right) \cup F_{0}(\Omega), \quad n \geqslant 2 .
$$

Consequently,

$$
W_{n+1}=W_{n} \cup \bigcup_{\left(j_{1}, \ldots, j_{n}\right) \in\{0, \ldots, L-1\}^{n}} F_{j_{1}} \ldots F_{j_{n}} F_{0}(\Omega), \quad n \geqslant 1,
$$

whence $W_{n} \subset W_{n+1}$.

By lemma 3.3, to show that $\operatorname{dim}_{H}(\Omega \backslash W)<d$, it suffices to demonstrate that there exists a positive constant $\delta=\delta(\Omega, \beta)>0$ such that given an arbitrary cube $\mathcal{C} \subset \Omega$, one can find a cube $\mathcal{C}_{0} \subset C$ such that $\mathcal{L}_{d}\left(\mathcal{C}_{0}\right) \geqslant \delta \mathcal{L}_{d}(\mathcal{C})$ and $\mathcal{C}_{0} \cap(\Omega \backslash W)=\emptyset$.

So we choose an arbitrary cube $\mathcal{C} \subset \Omega$ and denote the length of its edge by $\kappa$. Let $\xi$ denote the centre of $\mathcal{C}$; since $\Omega$ has no holes, $\xi=\lim _{r \rightarrow \infty} F_{j_{1}} \ldots F_{j_{r}}(\Omega)$ (the limit in the Hausdorff metric). Hence there exists a unique $N$ such that $F_{j_{1}} \ldots F_{j_{N-1}}(\Omega) \not \subset \mathcal{C}$ and $F_{j_{1}} \ldots F_{j_{N}}(\Omega) \subset \mathcal{C}$.

Note that since $\xi \in F_{j_{1}} \ldots F_{j_{N-1}}(\Omega)$ and $F_{j_{1}} \ldots F_{j_{N-1}}(\Omega) \not \subset \mathcal{C}$, we have

$$
\operatorname{diam} F_{j_{1}} \ldots F_{j_{N-1}}(\Omega) \geqslant \operatorname{dist}(\xi, \partial \mathcal{C})=\kappa / 2 \text {. }
$$

Put

$$
v=\frac{\operatorname{diam}(\Omega)}{\left(\mathcal{L}_{d}(\Omega)\right)^{1 / d}}
$$

Then

whence

$$
\begin{aligned}
\mathcal{L}_{d}(\mathcal{C})^{1 / d} & =\kappa \leqslant 2 \operatorname{diam} F_{j_{1}} \ldots F_{j_{N-1}}(\Omega)=2 \beta^{N-1} \operatorname{diam}(\Omega) \\
& =\frac{2 v}{\beta} \cdot \beta^{N}\left(\mathcal{L}_{d}(\Omega)\right)^{1 / d}=\frac{2 v}{\beta} \cdot\left(\mathcal{L}_{d}\left(F_{j_{1}} \ldots F_{j_{N-1}}(\Omega)\right)^{1 / d},\right.
\end{aligned}
$$

$$
\frac{\mathcal{L}_{d}\left(F_{j_{1}} \ldots F_{j_{N}}(\Omega)\right)}{\mathcal{L}_{d}(\mathcal{C})} \geqslant c>0
$$


where $c=(\beta / 2 v)^{d}$, i.e. $c$ depends only on the shape of $\Omega$ and on the contraction ratio, but not on $\mathcal{C}$ itself.

Put $\Omega_{0}=F_{j_{1}} \ldots F_{j_{N}} F_{0}(\Omega)$; by $(3.3), \mathcal{L}_{d}\left(\Omega_{0}\right) \geqslant \beta c \cdot \mathcal{L}_{d}(\mathcal{C})$. We can find a cube $\mathcal{C}_{1} \subset \Omega$ such that the ratio of their volumes equals $\gamma>0$. Since $\Omega_{0}$ is similar to $\Omega$, we put $\mathcal{C}_{0}=F_{j_{1}} \ldots F_{j_{N}} F_{0}\left(\mathcal{C}_{1}\right) \subset \Omega_{0}$ and obtain

$$
\mathcal{L}_{d}\left(\mathcal{C}_{0}\right) \geqslant \beta \gamma c \cdot \mathcal{L}_{d}(\mathcal{C}),
$$

where $\delta:=\beta \gamma c$ is independent of $\mathcal{C}$. Furthermore, $\Omega_{0}$ (and consequently, $\mathcal{C}_{0}$ ) has an empty intersection with $\Omega \backslash W_{N+1}$, whence $\mathcal{C}_{0} \cap(\Omega \backslash W)=\emptyset$ as well, and we are done.

\section{Theorem 3.5. Assume}

- $S_{\lambda}=\Omega$, i.e. there are no holes; and

- there exist $i, k \in \mathcal{A}$ such that a vertex of $f_{k}(\Omega)$ belongs to the interior of $f_{i}(\Omega)$.

Then $\mathcal{L}_{d}$-a.e. $\boldsymbol{x} \in \Omega$ has $2^{\aleph_{0}}$ distinct addresses, and the exceptional set $V_{\lambda}$ has Hausdorff dimension strictly less than $d$.

Remark 3.6. If $d \leqslant 2$, it suffices to assume that $f_{i}(\Omega) \cap f_{k}(\Omega)$ has a nonempty interior, since if two convex polygons (or intervals) intersect properly, then it is obvious that there exists a vertex of one which lies in the interior of the other. For $d \geqslant 3$ this is not always the case.

Proof. By our assumption, there exists $j \in \mathcal{A}$ such that $f_{k}\left(\boldsymbol{p}_{j}\right) \in \operatorname{int}\left(f_{i}(\Omega)\right)$. Hence there exists $\ell \in \mathbb{N}$ such that $f_{i} f_{j}^{\ell-1}(\Omega) \subset f_{i}(\Omega) \cap f_{k}(\Omega)$. Since $S_{\lambda}=\Omega$, lemma 2.5 implies that any $\boldsymbol{x} \in f_{i} f_{j}^{\ell-1}(\Omega)$ has at least two different addresses.

Put $L=m^{\ell}$ and define $\left\{F_{0}, \ldots, F_{L-1}\right\}=\left\{f_{i_{1}} \ldots f_{i_{\ell}} \mid\left(i_{1}, \ldots, i_{\ell}\right) \in \mathcal{A}^{\ell}\right\}$ with $F_{0}=f_{i} f_{j}^{\ell-1}$. By the above, each $\boldsymbol{x} \in F_{j_{1}} \ldots F_{j_{k-1}} F_{0} F_{j_{k+1}} \ldots F_{n}(\Omega)$ has at least two different addresses, whence $U_{\lambda} \subset \Omega \backslash W$, where $W=\bigcup_{n} W_{n}$ and $W_{n}$ is given by (3.2).

Hence by lemma $3.4, \operatorname{dim}_{H}\left(U_{\lambda}\right)<d$, whence by lemma $3.1, \operatorname{dim}_{H}\left(V_{\lambda}\right)<d$, which is the claim of the theorem.

Remark 3.7. If $\lambda$ is sufficiently close to the critical value $\lambda_{0}$ (see the previous section), then one could expect the exceptional set $V_{\lambda}$ to be countable (similarly to the one-dimensional case).

\subsection{Application: $\lambda$-expansions with deleted digits}

Expansions of real numbers in noninteger bases with deleted digits have been studied since the mid-1990s - see, e.g., [8, 12]. The model is as follows: assume $d=1$ and let $A=\left\{a_{1}, \ldots, a_{m}\right\} \subset \mathbb{R}$ be a 'digit' set with $a_{1}<\ldots<a_{m}$. Let $x \in \mathbb{R}$ have an expansion of the form

$$
x=\sum_{n=1}^{\infty} \varepsilon_{n} \lambda^{n}, \quad \varepsilon_{n} \in A, \quad n \geqslant 1 .
$$

It is obvious that $\lambda a_{1} /(1-\lambda) \leqslant x \leqslant \lambda a_{m} /(1-\lambda)$. Pedicini [11] has shown that if

$$
\max _{1 \leqslant j \leqslant m-1}\left(a_{j+1}-a_{j}\right)<\frac{\lambda\left(a_{m}-a_{1}\right)}{1-\lambda},
$$

then each $x \in\left[\lambda a_{1} /(1-\lambda), \lambda a_{m} /(1-\lambda)\right]$ has at least one expansion of the form (3.4). Note also that in the recent paper [2] the theory of random and greedy beta-expansions with deleted digits (under the assumption (3.5)) has been developed.

We apply our results from this and the previous section to obtain the following. 


\section{Proposition 3.8.}

(a) There exists $\lambda_{0}=\lambda_{0}\left(a_{1}, \ldots, a_{m}\right)<1$ such that for each $\lambda \in\left(\lambda_{0}, 1\right)$ any $x \in$ $\left(\lambda a_{1} /(1-\lambda), \lambda a_{m} /(1-\lambda)\right)$ has $2^{\aleph_{0}}$ expansions of the form (3.4).

(b) If the condition (3.5) is satisfied, Lebesgue-a.e. $x \in\left(\lambda a_{1} /(1-\lambda), \lambda a_{m} /(1-\lambda)\right)$ has $2^{\aleph_{0}}$ expansions of the form (3.4), and the exceptional set has Hausdorff dimension strictly less than 1.

Proof. Put

$$
f_{j}(x)=\lambda\left(x+a_{j}\right), \quad 1 \leqslant j \leqslant m .
$$

Then, as in the standard one-dimensional case (where $a_{1}=0, a_{2}=1$ ), we have by induction,

$$
f_{\varepsilon_{1}} \ldots f_{\varepsilon_{n}}\left(x_{0}\right)=\lambda^{n} x_{0}+\sum_{k=1}^{n} \varepsilon_{k} \lambda^{k}
$$

whence

$$
\lim _{n \rightarrow \infty} f_{\varepsilon_{1}} \ldots f_{\varepsilon_{n}}\left(x_{0}\right)=\sum_{n=1}^{\infty} \varepsilon_{n} \lambda^{n}
$$

for any $x_{0} \in \mathbb{R}$. Therefore, $x$ has an expansion of the form (3.4) if and only if $x \in S_{\lambda}$ for the IFS (3.6).

The condition (3.5) ensures that $S_{\lambda}=\Omega=\left[\lambda a_{1} /(1-\lambda), \lambda a_{m} /(1-\lambda)\right]$. To prove the first part of the proposition, note that (3.5) holds for all $\lambda$ sufficiently close to 1 so theorem 2.7 is applicable to any $x$ which lies in the interior of $\Omega$. (As $\partial \Omega=\left\{a_{1} /(1-\lambda), a_{m} /(1-\lambda)\right\}$.)

To prove the second part, we note that by (3.5),

$$
\left|f_{j}(\Omega)\right|=\frac{\lambda^{2}\left(a_{m}-a_{1}\right)}{1-\lambda}>\lambda\left(a_{j+1}-a_{j}\right), \quad 1 \leqslant j \leqslant m-1 .
$$

Hence

$$
\begin{aligned}
\sum_{j=1}^{m}\left|f_{j}(\Omega)\right| & =\sum_{j=1}^{m-1}\left|f_{j}(\Omega)\right|+\left|f_{m}(\Omega)\right| \\
& >\lambda\left(a_{m}-a_{1}\right)+\frac{\lambda^{2}\left(a_{m}-a_{1}\right)}{1-\lambda} \\
& =\frac{\lambda\left(a_{m}-a_{1}\right)}{1-\lambda}=|\Omega|,
\end{aligned}
$$

whence there exists $j \in\{1, \ldots, m-1\}$ such that $f_{j}(\Omega) \cap f_{j+1}(\Omega)$ has a nonempty interior. Thus, we can apply theorem 3.5 to this setting.

Remark 3.9. In her $\mathrm{PhD}$ dissertation, Lai [10] has proved a weaker version of the second claim of proposition 3.8.

Finally, we prove the following.

Lemma 3.10. Provided (3.5) is satisfied, the open set condition for the IFS (3.6) fails.

Proof. We have

$$
a_{m}-a_{1}=\sum_{j=1}^{m-1}\left(a_{j+1}-a_{j}\right)<\frac{\lambda(m-1)\left(a_{m}-a_{1}\right)}{1-\lambda},
$$

whence $\lambda>1 / m$, and we apply proposition 2.1 . 


\subsection{General theory: natural measure}

In the end of this section we would like to obtain a result similar to theorem 3.5 for a 'natural' measure on $S_{\lambda}$. Let $\left(p_{0}, p_{1}, \ldots, p_{m-1}\right)$ be a probability vector with $p_{j}>0$ for all $j$. The probabilistic IFS given by the $f_{i}$ and the $p_{i}$ is defined as follows: put $\Sigma=\mathcal{A}^{\mathbb{N}}$ and define $\rho$ as the product measure on $\Sigma$ with equal multipliers $\left(p_{0}, p_{1}, \ldots, p_{m-1}\right)$. Let the projection map $\pi: \Sigma \rightarrow \mathbb{R}^{d}$ be given by the formula

$$
\pi\left(i_{1}, i_{2}, \ldots\right):=\lim _{n \rightarrow+\infty} f_{i_{1}} f_{i_{2}} \ldots f_{i_{n}}(\mathbf{0}) .
$$

We define the measure $\mu$ on $S_{\lambda}$ as the push down measure $\pi(\rho)$. As is well known, $\operatorname{supp}(\mu)=S_{\lambda}$.

Proposition 3.11. Under the assumptions of theorem 3.5, $\mu$-a.e. $x \in \Omega$ has $2^{\aleph_{0}}$ distinct addresses.

Proof. Put $\boldsymbol{w}=i j^{\ell-1}$. By the Birkhoff ergodic theorem applied to the one-sided Bernoulli shift on the measure space $(\Sigma, \rho)$,

$$
\rho\left\{\left(i_{1}, i_{2}, \ldots\right) \in \Sigma \mid \exists k:\left(i_{k}, \ldots, i_{k+\ell-1}\right)=w\right\}=1,
$$

whence $\mu\left(U_{\lambda}\right)=0$, because $\boldsymbol{x} \in U_{\lambda}$ cannot have an address containing $\boldsymbol{w}$. All that is left is to show that $\mu\left(V_{\lambda}\right)=0$ as well.

In view of (3.1), it suffices to show that

$$
\mu\left(f_{i_{1}} \ldots f_{i_{n}}\left(U_{\lambda}\right)\right)=0, \quad \forall\left(i_{1}, \ldots, i_{n}\right) \in \mathcal{A}^{n} .
$$

Note that, as is well known (see, e.g., [3]), the self-similarity of the measure $\mu$ implies

$$
\mu(E)=\sum_{s=0}^{m-1} p_{s} \cdot \mu\left(f_{s}(E)\right),
$$

for any Borel set $E$. Hence by induction,

$$
\mu(E)=\sum_{\left(i_{1}, \ldots, i_{n}\right) \in \mathcal{A}^{n}} p_{i_{1}} \ldots p_{i_{n}} \cdot \mu\left(f_{i_{1}} \ldots f_{i_{n}}(E)\right),
$$

which implies (3.7).

Remark 3.12. In fact, to apply the ergodic theorem to the shift on $\Sigma$, all we need from $\rho$ is $p_{i}>0$ and $p_{j}>0$; the other components of the probability vector may equal zero.

The main problem for future study is to check whether in some cases of IFSs with holes an analogue of theorem 3.5 still holds. We plan to be study this question in our subsequent papers.

\section{Main example: triangle}

For the triangular case we give an explicit analogue of one-dimensional results mentioned in section 1. Following [1], we denote the set of uniqueness by $\mathcal{U}_{\lambda}$.

Theorem 4.1. Let $\lambda_{0} \approx 0.68233$ be the unique positive root of $x^{3}+x=1$. Then

(a) for $\lambda<\lambda_{0}$, the set of uniqueness $\mathcal{U}_{\lambda}$ is nonempty; and

(b) if $\lambda \in\left(\lambda_{0}, 1\right)$, then each $x \in \mathcal{S}_{\lambda} \backslash\left\{\boldsymbol{p}_{0}, \boldsymbol{p}_{1}, \boldsymbol{p}_{2}\right\}$ has $2^{\aleph_{0}}$ different addresses. 


\section{Proof.}

(1) Firstly, we introduce a convenient coordinate system for this case suggested in [1]. Without loss of generality, we may assume our triangle $\Delta$ to be equilateral. We now identify each point $\boldsymbol{x} \in \Delta$ with a triple $(x, y, z)$, where

$$
x=\operatorname{dist}\left(\boldsymbol{x},\left[\boldsymbol{p}_{1}, \boldsymbol{p}_{2}\right]\right), \quad y=\operatorname{dist}\left(\boldsymbol{x},\left[\boldsymbol{p}_{0}, \boldsymbol{p}_{2}\right]\right), \quad z=\operatorname{dist}\left(\boldsymbol{x},\left[\boldsymbol{p}_{0}, \boldsymbol{p}_{1}\right]\right),
$$

where $\left[\boldsymbol{p}_{i}, \boldsymbol{p}_{j}\right]$ is the edge containing $\boldsymbol{p}_{i}$ and $\boldsymbol{p}_{j}$. As is well known, $x+y+z$ equals the tripled radius of the inscribed circle, and we choose it to be equal to 1 . These coordinates are called barycentric. Henceforth we write each $\boldsymbol{x} \in \mathcal{S}_{\lambda}$ in barycentric coordinates.

It is shown in [1] that $(x, y, z) \in \mathcal{S}_{\lambda}$ if and only if there exist three 0 - 1 sequences $\left(a_{n}\right)_{0}^{\infty},\left(b_{n}\right)_{0}^{\infty}$ and $\left(c_{n}\right)_{0}^{\infty}$ such that

$$
\begin{aligned}
& x=(1-\lambda) \sum_{n=0}^{\infty} a_{n} \lambda^{n}, \\
& y=(1-\lambda) \sum_{n=0}^{\infty} b_{n} \lambda^{n}, \\
& x=(1-\lambda) \sum_{n=0}^{\infty} c_{n} \lambda^{n},
\end{aligned}
$$

with $a_{n}+b_{n}+c_{n}=1$ for all $n \geqslant 0$.

We claim that the point

$$
\pi(\lambda)=\left(\frac{\lambda^{2}}{1+\lambda+\lambda^{2}}, \frac{\lambda}{1+\lambda+\lambda^{2}}, \frac{1}{1+\lambda+\lambda^{2}}\right)
$$

belongs to $\mathcal{U}_{\lambda}$ provided $\lambda<\lambda_{0}$. To prove this, it suffices to demonstrate that the system of equations

$$
\begin{aligned}
& a_{0}+a_{1} \lambda+a_{2} \lambda^{2}+\cdots=\frac{\lambda^{2}}{1-\lambda^{3}}, \\
& b_{0}+b_{1} \lambda+b_{2} \lambda^{2}+\cdots=\frac{\lambda}{1-\lambda^{3}}, \\
& c_{0}+c_{1} \lambda+c_{2} \lambda^{2}+\cdots=\frac{1}{1-\lambda^{3}}
\end{aligned}
$$

has a unique solution $\left(a_{n}\right)_{0}^{\infty}=(001001 \ldots),\left(b_{n}\right)_{0}^{\infty}=(010010 \ldots),\left(c_{n}\right)_{0}^{\infty}=(100100 \ldots)$. Note first that $a_{0}$ cannot be equal to 1 nor can $b_{0}$, because $\lambda<\lambda_{0}$ implies $1>\frac{\lambda}{1-\lambda^{3}}>\frac{\lambda^{2}}{1-\lambda^{3}}$. Hence $a_{0}=b_{0}=0, c_{0}=1$. Similarly, $a_{1}=0$ for the same reason as $b_{0}$, and $c_{1}=0$ as well, because $\lambda>\frac{\lambda^{3}}{1-\lambda^{3}}$. Thus, $b_{1}=1$. Finally, $c_{2}$ and $b_{2}$ must be equal to 0 , whilst $a_{2}=1$.

Thus, we have

$$
\begin{aligned}
& a_{3}+a_{4} \lambda+a_{5} \lambda^{2}+\ldots=\frac{\lambda^{2}}{1-\lambda^{3}}, \\
& b_{3}+b_{4} \lambda+b_{5} \lambda^{2}+\ldots=\frac{\lambda}{1-\lambda^{3}}, \\
& c_{3}+c_{4} \lambda+c_{5} \lambda^{2}+\ldots=\frac{1}{1-\lambda^{3}},
\end{aligned}
$$

and we can continue the process ad infinitum. Therefore, each $a_{k}, b_{k}$ and $c_{k}$ is uniquely determined from the system of equations (4.2), whence $\pi(\lambda) \in \mathcal{U}_{\lambda}$. 


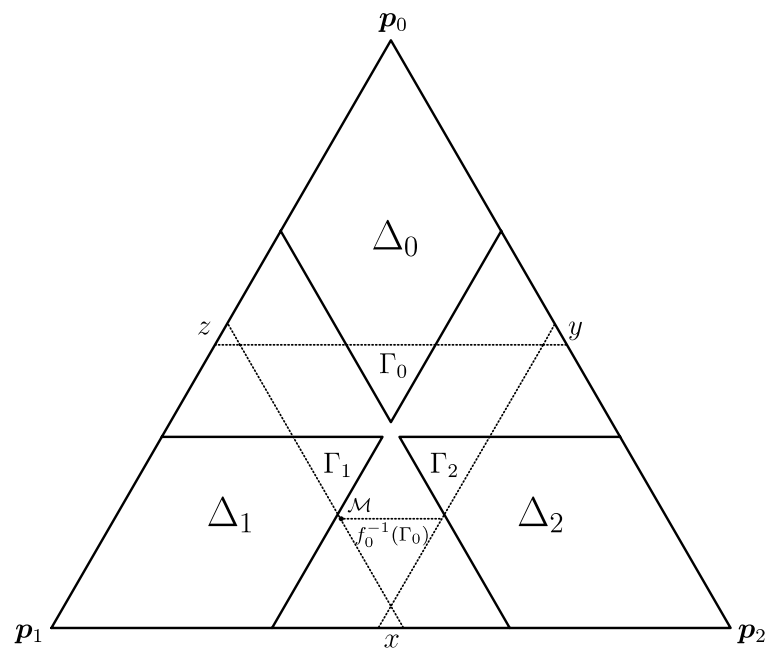

Figure 2. Triangular case: $f_{0}^{-1}\left(\Gamma_{0}\right) \cap\left(\bigcup_{i} \Delta_{i}\right)=\emptyset$.

(2) Suppose $\lambda>\lambda_{0}$. Following the argument of the proof of theorem 2.7, we introduce the sets

$$
\Delta_{i}:=\Delta \backslash \bigcup_{j \neq i} f_{j}(\Delta)
$$

(three rhombi). Thus, if $x \in \mathcal{U}_{\lambda}$, then necessarily $x \in \bigcup_{i} \Delta_{i}$. Fix $i \in\{0,1,2\}$; again, since the shift map on $\Delta_{i}$, i.e. $f_{i}^{-1}$, is expanding on $\Delta_{i} \backslash\left\{\boldsymbol{p}_{i}\right\}$, eventually $f_{i}^{-n}(\boldsymbol{x}) \in \Delta_{i}$ and $f_{i}^{-n-1}(\boldsymbol{x}) \notin \Delta_{i}$ for some $n \geqslant 0$, for any $\boldsymbol{x} \in \Delta_{i} \backslash\left\{\boldsymbol{p}_{i}\right\}$.

$$
\begin{aligned}
\text { If } f_{i}^{-n-1}(\boldsymbol{x}) \notin \bigcup_{j \neq i} \Delta_{j} \text {, then } \boldsymbol{x} \notin \mathcal{U}_{\lambda} \text {. Put } \\
\Gamma_{i}=f_{i}^{-1}\left(\Delta_{j}\right) \cap \Delta_{i}, \quad j \neq i .
\end{aligned}
$$

In view of the symmetry, the choice of $j \neq i$ is unimportant-see figure 2 . Thus, $\mathcal{U}_{\lambda} \neq \emptyset$ implies $\bigcup_{i} \Gamma_{i} \neq \emptyset$.

Note that the $\Gamma_{i}$ are equal for $i=0,1,2$, whence $\bigcup_{i} \Gamma_{i} \neq \emptyset \Leftrightarrow \Gamma_{0} \neq \emptyset$. The latter is in fact equivalent to $\lambda<1 / \sqrt{2}$. Indeed, we have in barycentric coordinates,

$$
\Gamma_{0}=\{x<(1-\lambda) / \lambda, y<1-\lambda, z<1-\lambda\} .
$$

An open triangle $\{x<a, y<b, z<c\}$ is nondegenerate if and only if $a+b+c>1$. Hence $(1-\lambda) / \lambda+2(1-\lambda)>1$, which is equivalent to $\lambda<1 / \sqrt{2}$. Thus, $\lambda \in\left(\lambda_{0}, 1 / \sqrt{2}\right)$.

Assume $\boldsymbol{x} \in \Gamma_{0} \cap \mathcal{U}_{\lambda}$; then $f_{0}^{-1}(\boldsymbol{x})$ has to intersect $\bigcup_{i} \Delta_{i}$. It is easy to check that $f_{0}^{-1}\left(\Gamma_{0}\right) \cap \Delta_{0}=\emptyset$, whence, in view of the symmetry, $f_{0}^{-1}\left(\Gamma_{0}\right) \cap \Delta_{1} \neq \emptyset$. We have

$$
\begin{aligned}
f_{0}^{-1}\left(\Gamma_{0}\right) & =\left\{x<\left(\frac{1-\lambda}{\lambda}\right)^{2}, y<\frac{1-\lambda}{\lambda}, z<\frac{1-\lambda}{\lambda}\right\}, \\
\Delta_{1} & =\{x<1-\lambda, y<1-\lambda\},
\end{aligned}
$$

and we claim that actually $f_{0}^{-1}\left(\Gamma_{0}\right)$ lies strictly on the right of $\Delta_{1}$ - see figure 2 .

Indeed, the coordinates of the point $\mathcal{M}$, the top left corner of the triangle $f_{0}^{-1}\left(\Gamma_{0}\right)$, are as follows: $\mathcal{M}\left(\left(\frac{1-\lambda}{\lambda}\right)^{2}, \frac{1-\lambda}{\lambda}, \frac{-1+\lambda+\lambda^{2}}{\lambda^{2}}\right)$, and we observe that the inequality $\frac{-1+\lambda+\lambda^{2}}{\lambda^{2}}>1-\lambda$ is equivalent to $\lambda>\lambda_{0}$. 
Thus, each $\boldsymbol{x} \in \Delta \backslash\left\{\boldsymbol{p}_{0}, \boldsymbol{p}_{1}, \boldsymbol{p}_{2}\right\}$ has at least two addresses of the form $\boldsymbol{x} \sim$ $\left(i_{1}, \ldots, i_{n}, i_{n+1}, \ldots\right)$ and $\boldsymbol{x} \sim\left(i_{1}, \ldots, i_{n}, j_{n+1}, \ldots\right)$ with $j_{n+1} \neq i_{n+1}$ and a 'compulsory' prefix $\left(i_{1}, \ldots, i_{n}\right)$, which may be empty (see figure 1). Hence $\boldsymbol{x}$ has $2^{\aleph_{0}}$ different addresses.

Remark 4.2. One can easily obtain from the proof of the previous theorem that $\mathcal{U}_{\lambda_{0}}=\emptyset$ as well, but in fact, the point $\pi\left(\lambda_{0}\right)=\left(\lambda_{0}^{4}, \lambda_{0}^{3}, \lambda_{0}^{2}\right)$ has only $\aleph_{0}$ different addresses. Thus, $\lambda_{0}$ is indeed the full analogue of the golden ratio for the triangular model. We leave the details as an exercise for the reader.

Remark 4.3. Note that if, like in the proof of theorem 2.7, $T_{\lambda}$ denotes the inverse of $\left\{f_{0}, f_{1}, f_{2}\right\}$ (well defined on $\bigcup_{i} \Delta_{i}$ ), then $\pi(\lambda)$ given by (4.1) is a period 3 point for $T_{\lambda}$, i.e. $T_{\lambda}^{3} \pi=\pi$. Another 3-cycle is generated by $\pi^{\prime}(\lambda)=\left(\frac{1}{1+\lambda+\lambda^{2}}, \frac{\lambda}{1+\lambda+\lambda^{2}}, \frac{\lambda^{2}}{1+\lambda+\lambda^{2}}\right)$, and we conjecture that if $\frac{2}{3} \leqslant \lambda<\lambda_{0}$, then $\bigcup_{i=0}^{2} \Gamma_{i} \cap \mathcal{U}_{\lambda}$ consists of just these 6 points. This would imply that $\mathcal{U}_{\lambda}$ is countable for this range of parameters.

A full 'triangular' analogue of theorem 1.3 is yet to be determined. In particular, what is the analogue of the Komornik-Loreti constant for the triangular case?

Note that for $\lambda=g=(\sqrt{5}-1) / 2$ the set $\mathcal{U}_{\lambda}$ is a continuum naturally isomorphic to the space of one-sided 0-1 sequences, and its Hausdorff dimension is - $\log 2 / \log g-$ see [1, theorem 6.4] and figure 5 therein.

\section{Acknowledgments}

The author is indebted to F Petrov for his generous help with the proof of proposition 2.4 and especially to the anonymous referee for many useful remarks and suggestions.

\section{References}

[1] Broomhead D, Montaldi J and Sidorov N 2004 Golden gaskets: variations on the Sierpinski sieve Nonlinearity 17 1455-80

[2] Dajani K and Kalle C 2007 Random beta-expansions with deleted digits Discrete. Contin. Dyn. Syst. 18 199-217

[3] Diaconis P and Freedman D 1999 Iterated random functions SIAM Rev. 41 45-76

[4] Eckhoff J 1993 Helly, Radon, and Caratheodory type theorems Handbook of Convex Geometry vol A, B (Amsterdam: North Holland) pp 389-448

[5] Erdős P, Joó I and Komornik V 1990 Characterization of the unique expansions $1=\sum_{i=1}^{\infty} q^{-n_{i}}$ and related problems Bull. Soc. Math. Fr. 118 377-90

[6] Falconer K 2003 Fractal Geometry: Mathematical Foundations and Applications (New York: Wiley)

[7] Glendinning P and Sidorov N 2001 Unique representations of real numbers in non-integer bases Math. Res. Lett. $8535-43$

[8] Keane M, Smorodinsky M and Solomyak B 1995 On the morphology of $\gamma$-expansions with deleted digits Trans. Am. Math. Soc. 347 955-66

[9] Komornik V and Loreti P 1998 Unique developments in non-integer bases Am. Math. Mon. 105 636-9

[10] Lai A-Ch 2006 Private communication

[11] Pedicini M 2005 Greedy expansions and sets with deleted digits Theor. Comput. Sci. 332 313-36

[12] Pollicott M and Simon K 1995 The Hausdorff dimension of $\lambda$-expansions with deleted digits Trans. Am. Math. Soc. 347 967-83

[13] Sidorov N 2003 Almost every number has a continuum of $\beta$-expansions Am. Math. Mon. 110 838-42

[14] Sidorov N 2003 Universal $\beta$-expansions Period. Math. Hung. 47 221-31 